\title{
Plasma Focus as a Powerful Hard X-Ray Source for Ultrafast Imaging of Moving Metallic Objects
}

\author{
V. Raspa ${ }^{(1,2)}$, L. Sigaut $^{(1)}$, R. Llovera ${ }^{(1,3)}$, P. Cobelli ${ }^{(1)}$, \\ P. Knoblauch ${ }^{(1)}$, R. Vieytes ${ }^{(1,4)}$, A. Clausse ${ }^{(5)}$, and C. Moreno ${ }^{(1)}$ \\ Interinstitutional Program of Dense Magnetized Plasmas - PLADEMA Argentina \\ (1) Departamento de Física, FCEyN-UBA, INFIP - CONICET \\ (2) Fellow of Fundación Antorchas \\ (3) Fellow of CONICET \\ (4) Escuela Superior Técnica Gral. Div. Manuel N. Savio, IESE \\ (5) Departamento de Computación y Sistemas, FCE - UNC, CNEA, CONICET
}

Received on 9 February, 2004; revised version received on 27 April, 2004

\begin{abstract}
Using high energy, pulsed X-rays, generated by a 4.7 kJ Plasma Focus operated with deuterium - argon admixtures, images of fast rotating metallic pieces were obtained. This illustrates the potential application of Plasma Focus devices for non invasive scanning of moving metallic objects. The samples for radiographic imaging were located outside the plasma focus chamber. High-sensitivity, fast-response commercial radiographic film was used as X-ray detector. The use of small doses of argon helps to enhance both the neutron production and the hard X-ray emission. By analyzing the mean attenuation of the studied radiation in different metals, an $\mathrm{X}$-ray effective energy of about $100 \mathrm{keV}$ was obtained.
\end{abstract}

\section{Introduction}

Plasma Focus (PF) are pulsed power devices that generate dense magnetized plasmas by means of electrical discharges in rarefied gases. These devices produce pulsed energetic beams of ions and electrons, a wide spectrum of electromagnetic radiation, and $2.45 \mathrm{MeV}$ neutrons (when filled with deuterium). The current basic and applied research interest of PF is related to dense plasma physics and innovative applications of pulsed radiation sources. The latter had attracted the attention of researchers during recent years, such as the use of ion beams for surface treatments [1,2], lithography by means of either soft X-rays or electron beams $[3,4]$, radiography [5,6], metal defectoscopy [7], and neutronic detection of hydrogen in samples $[8,9]$.

The fist part of this article shows how a small PF can be used as a hard X-ray source to take clear radiographs of fast moving metallic objects. Due to the extremely short duration of the X-ray emission (about $50 \mathrm{~ns}$ ), the exposure time is six orders of magnitude shorter than what can be reached with conventional sources. This characteristic, together with the degree of penetration of the associated radiation, contribute to produce high quality X-ray images of moving metallic objects.

In the second part, the characterization of the effective energy of the $\mathrm{X}$ radiation responsible for the radiographs is presented. The method is based on the analysis of the attenuation properties in different metallic samples.

\section{Experimental setup}

The experimental study was performed on the PF device GN1 detailed in [10-12]. The capacitance of the bank is $10.5 \mu \mathrm{F}$ and it is charged to $30 \mathrm{kV}(4.7 \mathrm{~kJ})$. The electrodes are a hollow copper anode and a squirrel-cage-like cathode made of bronze bars. Both electrodes are separated by a cylindrical Pyrex insulator, and located inside a cylindrical, $1 \mathrm{dm}^{3}$, stainless steel chamber (see Fig. 1). The chamber walls are $3 \mathrm{~mm}$ thick.

The device has a $1 \mathrm{~m}^{2}$ footprint and for optimal performance, the chamber is filled with deuterium mixed with $2.5 \%$ of argon. The optimal total pressure for X-ray production is 3.5 mbar.

The origin of the high energy X-ray emission is bremsstrahlung radiation produced during the collision of a collimated electron beam emitted by the focus, with the anode bottom. To enhance the hard X-ray emission a lead blanket was placed where the electron beam collides with the anode (see Fig. 1).

Figures 2 show two experimental setups used to obtain radiographic images of fast moving metallic objects. A stainless steel ball bearing (Fig. 2a) mounted on an axis was radiographied rotating at $2820 \mathrm{rpm}$. Fig. $2 \mathrm{~b}$ shows the setup to radiograph an aluminum turbine blade rotating at 6120 rpm. The radiographic film is located at $17 \mathrm{~cm}$ and $34 \mathrm{~cm}$ away from the PF chamber, for the ball bearing and the turbine, respectively. These distances were chosen to balance intensity and homogeneity over the film. A commercial $\mathrm{Cu}-$ rix Agfa $13 \times 18 \mathrm{~cm}$ film was used as in reference [7]. The hard X-ray production in every shot was monitored using a 
photomultiplier tube coupled with a NE 102A plastic scintillator placed $3.9 \mathrm{~m}$ away from the chamber.

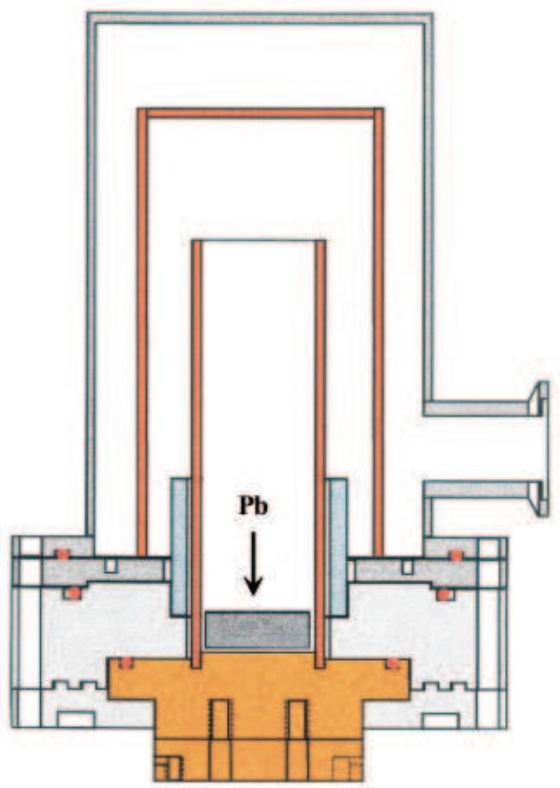

Figure 1. Sketch of GN1 Plasma Focus chamber.

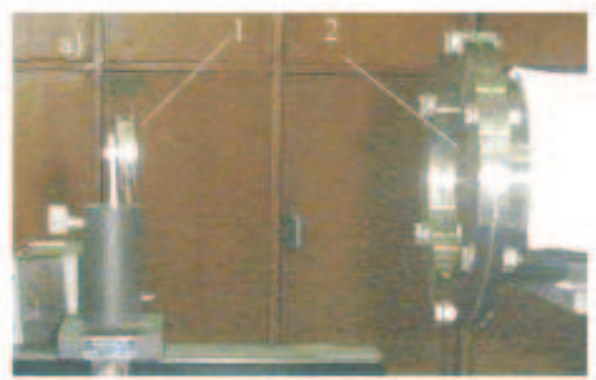

1) Ball bearing in the positioner

2) Discharges chamber

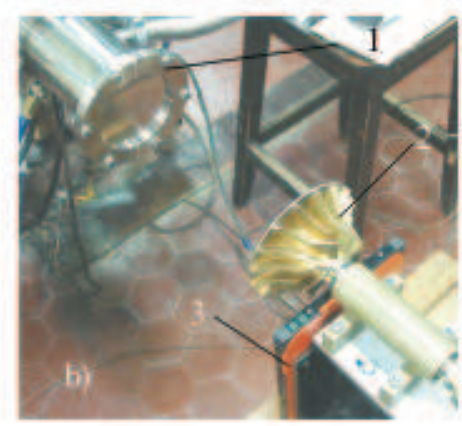

1) Discharges chamber

2) Turbine

3) Radiographic plate

Figure 2. Experimental setup used to obtain radiographs of (a) a ball bearing (b) a turbine.

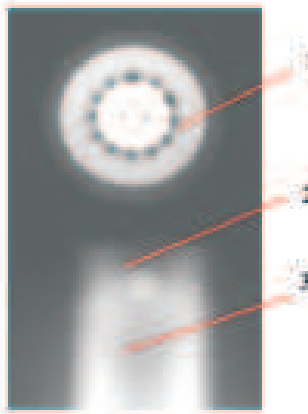

Static

$P P=-1.34 \mathrm{~V}$

$\mathrm{D}=17 \mathrm{~cm}, \mathrm{P}=4 \mathrm{mbar}$

$\mathrm{D}_{2}=100 \%, \omega=2820 \mathrm{rpm}$

1) Stainless steel balt bearing

2) Aluminium support

3) Metallic positioner

Figure 3. Radiographic images of a stainless steel ball bearing: static and rotating.

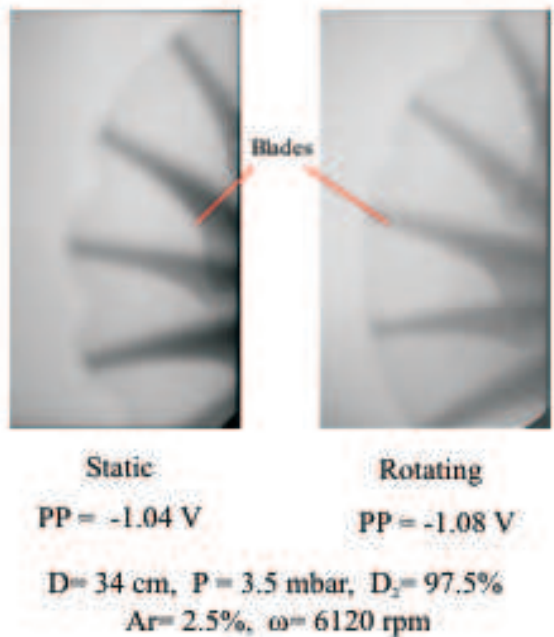

Figure 4. Radiographic images of an aluminum turbine: static and in fast rotation.

\section{Results}

\subsection{Hard X-ray imaging}

Figures 3 show the ball bearing images obtained with the object at rest and rotating. The darker regions observed in the images correspond to thickness differences. The inner balls can be seen in great detail in both images.

Figures 4 show the turbine negative images obtained with the object at rest and rotating. In both cases, the distribution of the blades can be clearly distinguished, including indentation marks on the border. 
The global contrast differences observed in Figs. 4 are due to intensity differences of the PF shots. The corresponding photomultiplier peak amplitudes (PP) are detailed in the figure caption. The global resolution of the digitized images was 300 dpi.

\subsection{Effective Energy}

To determine the effective energy of the radiation responsible for the radiographies shown above, the attenuation produced by metallic strips of different composition and thicknesses was measured and analyzed. Assuming a continuous energy spectrum, the total intensity of the incident beam is:

$$
I_{0}=\int_{0}^{\infty} S(E) d E
$$

where $S(E)$ is the spectral density and $E$ is the energy of the photons. In a first approximation, the intensity $I(x)$ of the beam after passing through a certain material is:

$$
I(x)=\int_{0}^{\infty} S(E) e^{-\mu(E) x} d E
$$

where $\mu(E)$ is the linear attenuation coefficient for that material and $x$ is the sample thickness. The relative attenuation due to the sample is:

$$
\frac{I(x)}{I_{0}}=\frac{\int_{0}^{\infty} S(E) e^{-\mu(E) x} d E}{\int_{0}^{\infty} S(E) d E}
$$

Equation (3) can be used to define an effective attenuation coefficient $\mu^{*} \equiv \mu\left(E^{*}\right)$ as the weighted average of the attenuation factor $\exp [-\mu(E) x]$, where the spectral density is the weighting coefficient:

$$
\frac{I(x)}{I_{0}}=e^{-\mu^{*} x}
$$

Measuring the attenuation ratio $I(x) / I_{0}$ for known sample thicknesses $x$ of the same material, it is possible to estimate $\mu^{*}$ for that material using equation (4). Once $\mu^{*}$ is known, $E^{*}$ can be estimated from the $\mu(E)$ relationship.

From the experimental point of view, $I(x) / I_{0}$ can be determined dividing the grey level of a radiograph with and without an attenuating sample, which in turn are numerically determined from the digitized images of the corresponding radiograph. Assuming a linear relation between the grey level, $N$, and the ratio $I(x) / I_{0}$ :

$$
N(x)=N_{\max }-\left(N_{\max }-N_{\min }\right) \frac{I(x)}{I_{0}}
$$

where $N_{\max }$ and $N_{\min }$ are the maximum and minimum grey levels of the radiograph. They correspond, respectively, to the minimum and maximum blackening of the film. For a
8 bits scanner, $N_{\max }$ is close to 255 and $N_{\min }$ to 0 . From equation (5):

$$
\frac{I(x)}{I_{0}}=\frac{N_{\max }-N(x)}{N_{\max }-N_{\min }}
$$

Equations (4) and (6) can be used to obtain $\mu^{*}$ for different materials. However equation (5) should be experimentally validated.

A series of radiographs of pure-metal samples (analytical quality) were obtained and analyzed. Table 1 details the sample materials and thicknesses, which were measured using a $0.01 \mathrm{~cm}$ precision caliper.

TABLE 1. Thicknesses of the metallic samples used in the attenuation study. Accuracy is $0.01 \mathrm{~cm}$.

\begin{tabular}{|c|c|}
\hline Material & Thicknesses $(\mathbf{c m})$ \\
\hline $\mathrm{Cd}$ & $0.11 ; 0.22 ; 0.30$ \\
$\mathrm{Cu}$ & $0.11 ; 0.22 ; 0.33 ; 0.44 ; 0.55$ \\
$\mathrm{Ni}$ & $0.16 ; 0.27 ; 0.43 ; 0.54$ \\
$\mathrm{Ti}$ & $0.36 ; 0.44 ; 0.56 ; 0.68$ \\
\hline
\end{tabular}

Figure 5 shows the measured dependence of $\ln \left[I(x) / I_{0}\right]$ with the sample thickness, $x$, for copper. The linearity that corroborates equation (5) in the studied range is clear. The slope of the line in Fig. 5 gives the value of the effective attenuation coefficient:

$$
\mu^{*} \text { (copper) }=(3.90 \pm 0.12) \mathrm{cm}^{-1}
$$

Using the tabulated attenuation properties for copper [13] one can determine the effective average energy of the $\mathrm{X}$ rays. Analogous procedures are followed with the attenuation data of the other materials. The resulting effective attenuation coefficients are detailed in Table 2. Among the studied materials, cadmium has the greater attenuation power, followed by copper and nickel. The average effective X-rays energy $E^{*}$ is around $100 \mathrm{keV}$.

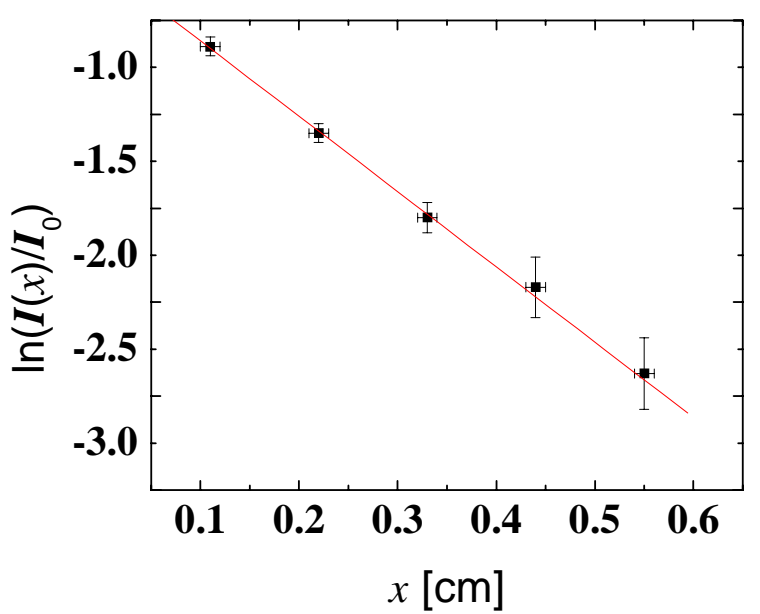

Figure 5. Graph of $\ln \left[I(x) / I_{0}\right]$ as function of the copper thickness for five samples. The continuous line corresponds to the linear adjustment of the data. 
The error bands in Table 2 come from the grey scale (digitalization of the image, discrete scale, etc.), and from the precision in $x$ measurements.

TABLE 2. Effective values of the attenuation coefficients and average $\mathrm{X}$-rays energy.

\begin{tabular}{|c|c|c|}
\hline Material & $\mu^{*}\left(\mathrm{~cm}^{-1}\right)$ & $E^{*}(\mathrm{keV})$ \\
\hline $\mathrm{Cd}$ & $9.48 \pm 0.13$ & $103 \pm 13$ \\
$\mathrm{Cu}$ & $3.90 \pm 0.12$ & $101 \pm 15$ \\
$\mathrm{Ni}$ & $3.35 \pm 0.04$ & $104 \pm 14$ \\
$\mathrm{Ti}$ & $1.16 \pm 0.03$ & $99 \pm 9$ \\
\hline
\end{tabular}

\section{Conclusions}

The images of metallic objects presented in this article demonstrate the feasibility of hard $\mathrm{X}$ ray emissions from small $\mathrm{PF}$ devices to obtain radiographs of rapidly rotating industrial samples. This in turn open the way toward innovative applications of plasma technology in non-destructive testing of materials, such as the detection of dynamic tensions on defects.

The study of the spectrum of the $\mathrm{X}$ radiation coming from the chamber shows energy values in agreement with previous measurement using different techniques [14,15]. The procedure presented in the present study is interesting, simple and not expensive, which can facilitate the research in small laboratories in developing countries. In particular, an interesting issue to explore is the dependence of the $\mathrm{X}$-rays energy with the PF design, such as anode material, charging voltage, filling gas and operating pressure.

\section{Acknowledgements}

This research was supported by Fundación Antorchas (Project 1406824), the IAEA (TC Proj. ARG1026), PLADEMA and UBA (X074).

\section{References}

[1] J. Feugeas, E. Llonch, C. de González, and G. Galambos, J. Appl. Phys. 64 (5), 2648 (1988).
[2] H. Kelly, A. Lepone, A. Márquez, D. Lamas, and C. Oviedo, Plasma Sources Sci. Technol. 5 (4), 704 (1996).

[3] S. Lee, P. Lee, G. Zhang, X. Feng, V. Gribkov, M. Liu, A. Serban, and T. Wong, IEEE Trans. on Plasma Sci. 26 (4), 1119 (1998).

[4] P. Lee, X. Feng, G. Zhang, M. Liu, and S. Lee, Plasma Sources Sci. Technol. 6 (3), 343 (1997).

[5] F. Castillo, M. Milanese, R. Moroso, J. Pouzo, and M. Santiago, IEEE Trans on Plasma Sci. 29 (6), 921 (2001).

[6] F. Castillo, J. J. E. Herrera, J. Rangel, A. Alfaro, M. A. Maza, V. Sakaguchi, G. Espinosa, and J. I. Golzarri, Braz. J. Phys. 32, 3 (2002).

[7] C. Moreno, A. Clausse, J. Martínez, R. Llovera, and A. Tartaglione, Nukleonika, 46 (1), 33 (2001).

[8] J. Pouzo, M. Milanese, and R. Moroso, AIP Conference Proc. 669 (1), 277 (2003).

[9] C. Moreno, A. Clausse, J. Martínez, R. Llovera, A. Tartaglione, M. Vénere, R. Barbuzza, and M. del Fresno, IX Latin American Workshop on Plasma Physics, La Serena, Chile, AIP Conference Proc., 563, H. Chuaqui and M. Favre Eds, 300-305, Nov (2000).

[10] C. Moreno, A. Clausse, H. Bruzzone, J. Martínez, R. Llovera, and A. Tartaglione IX Latin American Workshop on Plasma Physics, La Serena, Chile, AIP Conference Proc., 563, H. Chuaqui and M. Favre Eds, 276-281, Nov (2000).

[11] C. Moreno, M. Vénere, R Barbuzza, M. Del Fresno, R. Ramos, H. Bruzzone, P. Florido, J. González, and A. Clausse, Braz. J. Phys. 32, 20 (2002).

[12] C. Moreno, H. Bruzzone, J. Martínez, and A. Clausse, IEEE Trans. on Plasma Sci. 28 (5), 1735 (2000).

[13] J. Hubbell and S. Seltzer, X-Rays attenuation coefficients. Ionizing radiation division, Physics Laboratory. National Institute of Standards and Technology, Gaithersburg, MD, USA, (1996).

[14] J. Mather. Dense Plasma Focus. In: Methods of Experimental Physics 9 B, edited by R. Lovberg and H. Griem, Academic Press, New York and London, 187 (1971).

[15] P. Choi, C. Deeney, H. Herold, and C. Wong, Laser and Particle Beams, 8 (3), 469 (1990). 